\title{
COMPLEX RANDOM MATRICES AND RAYLEIGH CHANNEL CAPACITY*
}

\author{
T. RATNARAJAH ${ }^{\dagger}$, R. VAILLANCOURT ${ }^{\dagger}$, AND M. ALVO $^{\dagger}$
}

\begin{abstract}
The eigenvalue densities of complex central Wishart matrices are investigated with the objective of studying an open problem in channel capacity. These densities are represented by complex hypergeometric functions of matrix arguments, which can be expressed in terms of complex zonal polynomials. The connection between the complex Wishart matrix theory and information theory is given. This facilitates the evaluation of the most important information-theoretic measure, the so-called channel capacity. In particular, the capacity of multiple input, multiple output (MIMO) Rayleigh distributed channels are fully investigated. We consider both correlated and uncorrelated channels and derive the corresponding channel capacity formulas. It is shown how the channel correlation degrades the capacity of the communication system.
\end{abstract}

Keywords. Complex random matrix, complex Wishart matrix, complex zonal polynomials, complex hypergeometric functions, Rayleigh distributed MIMO channel, channel capacity

AMS(MOS) subject classification. 94A15, 94A05, 60E05, 62H10

1. Introduction. Let an $n \times m$ complex Gaussian random matrix $\mathbf{A}$ be distributed as $\mathbf{A} \sim \mathcal{C} N\left(\mathbf{0}, I_{n} \otimes \Sigma\right)$ with mean $\mathcal{E}\{\mathbf{A}\}=\mathbf{0}$ and covariance $\operatorname{cov}\{\mathbf{A}\}=I_{n} \otimes \Sigma$. Then the matrix $\mathbf{W}=\mathbf{A}^{H} \mathbf{A}$ is called a complex central Wishart matrix and its distribution is denoted by $\mathcal{C} W_{m}(n, \Sigma)$.

In this paper, we investigate the densities of the eigenvalues of complex central Wishart matrices and their applications to multiple input, multiple output (MIMO) channel capacity. We consider that the elements of random matrices are complex Gaussian distributed with zero mean and arbitrary covariance matrices. This will enable us to consider the beautiful but difficult theory of complex zonal polynomials (also called Schur polynomials [11]), which are symmetric polynomials in the eigenvalues of a complex matrix [14]. Complex zonal polynomials enable us to represent the densities of the eigenvalues of these complex Wishart matrices as infinite series.

The theory of these complex Wishart matrices is used to evaluate the capacity of MIMO wireless communication systems. Note that the capacity of a communication channel expresses the maximum rate at which information can be reliably conveyed by the channel [1]. In a wireless communication system, data is delivered from a transmitter to a receiver using radio waves or other electromagnetic waves. The waves, however, may be reflected off objects in the environment and scattered randomly while

\footnotetext{
*Received on April 30, 2003; accepted for publication on July 1, 2003. This work was partially supported by the Natural Sciences and Engineering Research Council of Canada.

${ }^{\dagger}$ Department of Mathematics and Statistics, University of Ottawa, 585 King Edward Ave, Ottawa ON K1N 6N5 Canada.

${ }^{\ddagger}$ Corresponding author, E-mail: remi@uottawa.ca, tel. 1-613-562-5864, fax 1-613-562-5776
} 
propagating from the transmitter to the receiver. Therefore, transmitted signals are attenuated and phase shifted during the transmission. This channel effect can be modeled by complex channel coefficients. A MIMO channel can be represented by an $n_{r} \times n_{t}$ complex random matrix $\mathbf{H} \sim \mathcal{C} N\left(\mathbf{0}, I_{n_{r}} \otimes \Sigma\right)$, where $n_{t}$ and $n_{r}$ are the number of inputs (or transmitters) and outputs (or receivers) of the wireless communication system. If $\Sigma=\sigma^{2} I_{n_{t}}$ then the channel is called an uncorrelated Rayleigh distributed channel, otherwise it is called a correlated Rayleigh distributed channel.

Recently, industrial researchers have exploited the use of MIMO systems to meet the demand for higher bit rates in wireless communications. These studies show that MIMO systems increase capacity significantly over single input, single output (SISO) systems. For example, when $n=\min \left\{n_{t}, n_{r}\right\}$, a MIMO uncorrelated Rayleigh distributed channel achieves almost $n$ more bits per hertz for every 3 -dB increase in signal-to-noise ratio (SNR) compared to a SISO system, which achieves only one additional bit per hertz for every 3-dB increase in SNR [18]. However, the channel coefficients from different transmitter antennas to a single receiver antenna can be correlated. This channel correlation degrades the capacity [4], [17]. The channel correlation depends on the physical parameters of a MIMO system and the scatterer characteristics. The physical parameters include the antenna arrangement and spacing, the angle spread, the angle of arrival, etc. One of the objectives of this paper is to evaluate this capacity degradation by deriving closed form ergodic capacity formulas for correlated channels and their numerical evaluation.

This paper is organized as follows. Section 2 provides the necessary tools for deriving the distribution theory and channel capacity. Complex central Wishart matrices are studied in Section 3. The capacity of MIMO channels is formulated in Section 4 and the computational methods are given in Section 5.

2. Preliminary tools. In this section we present tools that will be used in the sequel.

2.1. Complex zonal polynomials. First, we define the multivariate hypergeometric coefficients $[a]_{\kappa}^{(\alpha)}$ which frequently occur in integrals involving zonal polynomials. Let $\kappa=\left(k_{1}, \ldots, k_{m}\right)$ be a partition of the integer $k$ with $k_{1} \geq \cdots \geq k_{m} \geq 0$ and $k=k_{1}+\cdots+k_{m}$. Then [2]

$$
[a]_{\kappa}^{(\alpha)}=\prod_{i=1}^{m}\left(a-\frac{1}{\alpha}(i-1)\right)_{k_{i}}
$$

where $(a)_{k}=a(a+1) \cdots(a+k-1)$ and $\alpha=1$ for complex and $\alpha=2$ for real multivariate hypergeometric coefficients, respectively. In this paper we only consider the complex case; therefore, for notational simplicity we drop the superscript [9], i.e.,

$$
[a]_{\kappa}:=[a]_{\kappa}^{(1)}=\prod_{i=1}^{m}(a-i+1)_{k_{i}} .
$$


The complex zonal polynomial (also called Schur polynomial [11]) of a complex matrix $X$ is defined in [8] by

$$
C_{\kappa}(X)=\chi_{[\kappa]}(1) \chi_{[\kappa]}(X)
$$

where $\chi_{[\kappa]}(1)$ is the dimension of the representation $[\kappa]$ of the symmetric group given by

$$
\chi_{[\kappa]}(1)=k ! \frac{\prod_{i<j}^{m}\left(k_{i}-k_{j}-i+j\right)}{\prod_{i=1}^{m}\left(k_{i}+m-i\right) !}
$$

and $\chi_{[\kappa]}(X)$ is the character of the representation $[\kappa]$ of the linear group given as a symmetric function of the eigenvalues $\lambda_{1}, \ldots, \lambda_{m}$ of $X$ by

$$
\chi_{[\kappa]}(X)=\frac{\operatorname{det}\left[\left(\lambda_{i}^{k_{j}+m-j}\right)\right]}{\operatorname{det}\left[\left(\lambda_{i}^{m-j}\right)\right]} .
$$

Note that both the real and complex zonal polynomials are particular cases of the (general $\alpha$ ) Jack polynomials $C_{\kappa}^{(\alpha)}(X)$. See [2] for details. Again $\alpha=1$ for complex and $\alpha=2$ for real zonal polynomials, respectively. For the same reason as before, we shall drop the superscript of Jack polynomials, as was done in (1), i.e., $C_{\kappa}(X):=$ $C_{\kappa}^{(1)}(X)$.

The following basic properties are given in [8]:

$$
(\operatorname{tr} X)^{k}=\sum_{\kappa} C_{\kappa}(X)
$$

and

$$
\int_{U(m)} C_{\kappa}\left(A X B X^{H}\right)(d X)=\frac{C_{\kappa}(A) C_{\kappa}(B)}{C_{\kappa}\left(I_{m}\right)},
$$

where $(d X)$ is the invariant measure on the unitary group $U(m)$, normalized to make the total measure unity, and

$$
C_{\kappa}\left(I_{m}\right)=2^{2 k} k !\left[\frac{1}{2} m\right]_{\kappa} \frac{\prod_{i<j}^{r}\left(2 k_{i}-2 k_{j}-i+j\right)}{\prod_{i=1}^{r}\left(2 k_{i}+r-i\right) !},
$$

where

$$
\left[\frac{1}{2} m\right]_{\kappa}=\prod_{i=1}^{r}\left(\frac{1}{2}(m-i+1)\right)_{k_{i}} .
$$

Note that the partition $\kappa$ of $k$ has $r$ nonzero parts.

2.2. Complex hypergeometric functions. The probability distributions of random matrices are often derived in terms of hypergeometric functions of matrix arguments. The following definitions of hypergeometric functions with a single and double matrix argument are due to Constantine [5] and Baker [2]. 
DeFINITION 1. The hypergeometric function of one complex matrix is defined as

$$
{ }_{p} F_{q}^{(\alpha)}\left(a_{1}, \ldots, a_{p} ; b_{1}, \ldots, b_{q} ; X\right)=\sum_{k=0}^{\infty} \sum_{\kappa} \frac{\left[a_{1}\right]_{\kappa}^{(\alpha)} \cdots\left[a_{p}\right]_{\kappa}^{(\alpha)}}{\left[b_{1}\right]_{\kappa}^{(\alpha)} \cdots\left[b_{q}\right]_{\kappa}^{(\alpha)}} \frac{C_{\kappa}^{(\alpha)}(X)}{k !},
$$

where $X \in \mathbb{C}^{m \times m}$ and $\left\{a_{i}\right\}_{i=1}^{p}$ and $\left\{b_{i}\right\}_{i=1}^{q}$ are arbitrary complex numbers. Note that $\sum_{\kappa}$ denotes summation over all partitions $\kappa$ of $k$ and $\alpha=1$ and 2 for complex and real hypergeometric functions, respectively.

In this paper we consider only the complex case, and hence, we shall drop the superscript, i.e., ${ }_{p} F_{q}:={ }_{p} F_{q}^{(1)}$. Note that none of the parameters $b_{i}$ is allowed to be zero or an integer or half-integer $\leq(m-1) / 2$. Otherwise some of the terms in the denominator will be zero [14].

REMARK 1. The convergence of (5) is as follows [14]:

(i) If $p \leq q$, then the series converges for all $X$.

(ii) If $p=q+1$, then the series converges for $\sigma(X)<1$, where the spectral radius $\sigma(X)$ of $X$ is the maximum of the absolute values of the eigenvalues of $X$.

(iii) If $p>q+1$, then the series diverges for all $X \neq 0$, unless it terminates. Note that the series terminates when some of the numerators $\left[a_{j}\right]_{\kappa}$ in the series vanish.

Special cases are

$$
{ }_{0} F_{0}(X)=\operatorname{etr}(X), \quad{ }_{1} F_{0}(a ; X)=\operatorname{det}(I-X)^{-a},
$$

and

$$
{ }_{0} F_{1}\left(n ; Z Z^{H}\right)=\int_{U(n)} \operatorname{etr}(Z E+\overline{Z E})(d E)
$$

where $Z$ is an $m \times n$ complex matrix with $m \leq n$, etr denotes the exponential of the trace, $\operatorname{etr}(\cdot)=\exp (\operatorname{tr}(\cdot))$ and $\overline{Z E}$ denotes the complex conjugate of $Z E$.

DeFINITION 2. The complex hypergeometric function of two complex matrices is defined by

$$
{ }_{p} F_{q}\left(a_{1}, \ldots, a_{p} ; b_{1}, \ldots, b_{q} ; X, Y\right)=\sum_{k=0}^{\infty} \sum_{\kappa} \frac{\left[a_{1}\right]_{\kappa} \cdots\left[a_{p}\right]_{\kappa}}{\left[b_{1}\right]_{\kappa} \cdots\left[b_{q}\right]_{\kappa}} \frac{C_{\kappa}(X) C_{\kappa}(Y)}{k ! C_{\kappa}\left(I_{m}\right)},
$$

where $X, Y \in \mathbb{C}^{m \times m}$.

The splitting formula is

$$
\int_{U(m)}{ }_{p} F_{q}\left(A E B E^{H}\right)(d E)={ }_{p} F_{q}(A, B) .
$$

3. The complex central Wishart matrix. In this section, we describe the complex central Wishart distribution and give the joint eigenvalue density of the complex central Wishart matrix. From this density we derive a single unordered eigenvalue density of the complex central Wishart matrix. 
The definition of the complex central Wishart distribution is as follows.

Definition 3. Let $\mathbf{W}=\mathbf{A}^{H} \mathbf{A}$, where the $n \times m$ matrix $\mathbf{A}$ is distributed as $\mathbf{A} \sim \mathcal{C} N\left(\mathbf{0}, I_{n} \otimes \Sigma\right)$. Then $\mathbf{W}$ is said to have the complex central Wishart distribution with $n$ degrees of freedom and covariance matrix $\Sigma$, denoted by $\mathbf{W} \sim \mathcal{C} W_{m}(n, \Sigma)$.

Let $\mathbf{W} \sim \mathcal{C} W_{m}(n, \Sigma)$ with $n \geq m$. Then the density of $\mathbf{W}$ is given by

$$
f(W)=\frac{1}{\mathcal{C} \Gamma_{m}(n)(\operatorname{det} \Sigma)^{n}} \operatorname{etr}\left(-\Sigma^{-1} W\right)(\operatorname{det} W)^{n-m},
$$

where $\mathcal{C} \Gamma_{m}(n)$ denotes the complex multivariate gamma function,

$$
\mathcal{C} \Gamma_{m}(n)=\pi^{m(m-1) / 2} \prod_{k=1}^{m} \Gamma(n-k+1)
$$

Next, we consider the eigenvalue density of a complex Wishart matrix.

Proposition 1. Let $\mathbf{W}$ be an arbitrary $m \times m$ positive definite complex random matrix with distribution function $f(W)$. Then the joint density function of the eigenvalues, $\lambda_{1}>\lambda_{2}>\cdots>\lambda_{m}>0$, of $\mathbf{W}$ is

$$
f(\Lambda)=\frac{\pi^{m(m-1)}}{\mathcal{C} \Gamma_{m}(m)} \prod_{k<l}^{m}\left(\lambda_{k}-\lambda_{l}\right)^{2} \int_{U(m)} f\left(E \Lambda E^{H}\right)(d E),
$$

where $\Lambda=\operatorname{diag}\left(\lambda_{1}, \ldots, \lambda_{m}\right)$ and $W=E \Lambda E^{H}$ is the eigendecomposition of $W$.

The following proposition gives the joint density of the eigenvalues of a complex Wishart matrix [8].

Proposition 2. Suppose that $n>m-1$ and consider the $m \times m$ positive definite Hermitian matrix $\mathbf{W} \sim \mathcal{C} W_{m}(n, \Sigma)$. Then the joint density of the eigenvalues, $\lambda_{1}>\lambda_{2}>\cdots>\lambda_{m}>0$, of $\mathbf{W}$ is

$$
\begin{aligned}
f(\Lambda)= & \frac{\pi^{m(m-1)}(\operatorname{det} \Sigma)^{-n}}{\mathcal{C} \Gamma_{m}(m) \mathcal{C} \Gamma_{m}(n)} \\
& \times \prod_{k=1}^{m} \lambda_{k}^{n-m} \prod_{k<l}^{m}\left(\lambda_{k}-\lambda_{l}\right)^{2} \int_{U(m)} \operatorname{etr}\left(-\Sigma^{-1} E \Lambda E^{H}\right)(d E),
\end{aligned}
$$

where $\Lambda=\operatorname{diag}\left(\lambda_{1}, \ldots, \lambda_{m}\right)$. Moreover,

$$
\begin{aligned}
\int_{U(m)} \operatorname{etr}\left(-\Sigma^{-1} E \Lambda E^{H}\right)(d E) & =\int_{U(m)}{ }_{0} F_{0}\left(-\Sigma^{-1} E \Lambda E^{H}\right)(d E) \\
& ={ }_{0} F_{0}\left(-\Sigma^{-1}, \Lambda\right) \\
& =\sum_{k=0}^{\infty} \sum_{\kappa} \frac{C_{\kappa}\left(-\Sigma^{-1}\right) C_{\kappa}(\Lambda)}{k ! C_{\kappa}\left(I_{m}\right)}
\end{aligned}
$$

Proof. By substituting the complex Wishart density (6) into (7) and noting that $\operatorname{det} W=\operatorname{det} E \Lambda E^{H}=\prod_{k=1}^{m} \lambda_{k}$ we obtain (8). 
Note that the integral in (8) depends on the population covariance matrix $\Sigma$ only through its eigenvalues $v_{1}, \ldots, v_{m}$. This can be seen by writing $\Sigma=F \Upsilon F^{H}$, where $F \in U(m)$ and $\Upsilon=\operatorname{diag}\left(v_{1}, \ldots, v_{m}\right)$. Now we have

$$
\begin{aligned}
\int_{U(m)} \operatorname{etr}\left(-\Sigma^{-1} E \Lambda E^{H}\right)(d E) & =\int_{U(m)} \operatorname{etr}\left(-F \Upsilon^{-1} F^{H} E \Lambda E^{H}\right)(d E) \\
& =\int_{U(m)} \operatorname{etr}\left(-\Upsilon^{-1} F^{H} E \Lambda E^{H} F\right)(d E) \\
& =\int_{U(m)} \operatorname{etr}\left(-\Upsilon^{-1} \widehat{E} \Lambda \widehat{E}^{H}\right)(d \widehat{E}) \\
& =\int_{U(m)}{ }_{0} F_{0}\left(-\Upsilon^{-1} \widehat{E} \Lambda \widehat{E}^{H}\right)(d \widehat{E}) \\
& ={ }_{0} F_{0}\left(-\Upsilon^{-1}, \Lambda\right) \\
& =\sum_{k=0}^{\infty} \sum_{\kappa} \frac{C_{\kappa}\left(-\Upsilon^{-1}\right) C_{\kappa}(\Lambda)}{k ! C_{\kappa}\left(I_{m}\right)}
\end{aligned}
$$

where $\widehat{E}=F^{H} E \in U(m)$ and $(d E)=(d \widehat{E})$. This was observed in [14]. In general, the integral in (8) is not easy to evaluate. An infinite series representation for this integral in terms of complex zonal polynomials is shown in (9).

Note that the density given in (8) is an ordered eigenvalue density and the unordered eigenvalue density is obtained by dividing (8) by $m$ !, i.e.,

$$
\frac{\pi^{m(m-1)}(\operatorname{det} \Sigma)^{-n}}{m ! \mathcal{C} \Gamma_{m}(m) \mathcal{C} \Gamma_{m}(n)} \prod_{k=1}^{m} \lambda_{k}^{n-m} \prod_{k<l}^{m}\left(\lambda_{k}-\lambda_{l}\right)^{2}{ }_{0} F_{0}\left(-\Upsilon^{-1}, \Lambda\right) .
$$

Let $\Upsilon^{-1}=\operatorname{diag}\left(a_{1}, \ldots, a_{m}\right)$. Then ${ }_{0} F_{0}\left(-\Upsilon^{-1}, \Lambda\right)$ can be written [10] as

$$
{ }_{0} F_{0}\left(-\Upsilon^{-1}, \Lambda\right)=\frac{\mathcal{C} \Gamma_{m}(m) \operatorname{det}\left[\left(\exp \left(-a_{i} \lambda_{j}\right)\right)\right]}{\pi^{m(m-1) / 2} \prod_{k<l}^{m}\left(\lambda_{k}-\lambda_{l}\right) \prod_{k<l}^{m}\left(a_{l}-a_{k}\right)} .
$$

A single unordered eigenvalue density is given by the following theorem to be used for computing the correlated MIMO channel capacity in Section 5 .

Theorem 1. Suppose that $n>m-1$ and consider the $m \times m$ positive definite Hermitian matrix $\mathbf{W} \sim \mathcal{C} W_{m}(n, \Sigma)$. Then the single unordered eigenvalue density $f\left(\lambda_{1}\right)$ of $\mathbf{W}$ is given by

$$
\begin{aligned}
& f\left(\lambda_{1}\right)=\frac{\pi^{m(m-1) / 2} \prod_{k=1}^{m} a_{k}^{n}}{m ! \mathcal{C} \Gamma_{m}(n) \prod_{k<l}^{m}\left(a_{l}-a_{k}\right)} \\
& \times \int\left\{\widetilde{\sum_{i}}(-1)^{\operatorname{per}\left(i_{1}, \ldots, i_{m}\right)} \exp \left(\sum_{j=1}^{m}-a_{i_{j}} \lambda_{j}\right)\right\} \\
& \times\left\{\widetilde{\sum_{k}}(-1)^{\operatorname{per}\left(k_{1}, \ldots, k_{m}\right)} \prod_{l=1}^{m} \lambda_{l}^{n-m+k_{l}}\right\} \bigwedge_{k=2}^{m} d \lambda_{k},
\end{aligned}
$$

where $\widetilde{\sum}_{i}$ denotes summation over all permutations $\left(i_{1}, \ldots, i_{m}\right)$ of $(1, \ldots, m), \widetilde{\sum}_{k}$ denotes summation over all permutations $\left(k_{1}, \ldots, k_{m}\right)$ of $(0, \ldots, m-1)$, and $\operatorname{per}\left(k_{1}, \ldots\right.$, 
$\left.k_{m}\right)$ is 0 or 1 depending on the permutation being even or odd. Similarly for $\operatorname{per}\left(i_{1}, \ldots\right.$, $\left.i_{m}\right)$.

Proof. The single unordered eigenvalue density is obtained by substituting (11) in (10) and integrating with respect to $\lambda_{2}, \ldots, \lambda_{m}$, i.e.,

$$
\begin{aligned}
f\left(\lambda_{1}\right)= & \frac{\pi^{m(m-1) / 2} \prod_{k=1}^{m} a_{k}^{n}}{m ! \mathcal{C} \Gamma_{m}(n) \prod_{k<l}^{m}\left(a_{l}-a_{k}\right)} \\
& \times \int \operatorname{det}\left[\left(\exp \left(-a_{i} \lambda_{j}\right)\right)\right] \prod_{k<l}^{m}\left(\lambda_{k}-\lambda_{l}\right) \prod_{k=1}^{m} \lambda_{k}^{n-m} \bigwedge_{k=2}^{m} d \lambda_{k} .
\end{aligned}
$$

The integrand in (13) can be written as

$$
\begin{aligned}
& \operatorname{det}\left[\left(\exp \left(a_{i} \lambda_{j}\right)\right)\right] \prod_{k<l}^{m}\left(\lambda_{k}-\lambda_{l}\right) \prod_{k=1}^{m} \lambda_{k}^{n-m} \\
& =\operatorname{det}\left[\begin{array}{ccc}
e^{-a_{1} \lambda_{1}} & \cdots & e^{-a_{1} \lambda_{m}} \\
e^{-a_{2} \lambda_{1}} & \cdots & e^{-a_{2} \lambda_{m}} \\
\vdots & \vdots & \vdots \\
e^{-a_{m} \lambda_{1}} & \cdots & e^{-a_{m} \lambda_{m}}
\end{array}\right] \operatorname{det}\left[\begin{array}{ccc}
1 & \cdots & 1 \\
\lambda_{1} & \cdots & \lambda_{m} \\
\vdots & \vdots & \vdots \\
\lambda_{1}^{m-1} & \cdots & \lambda_{m}^{m-1}
\end{array}\right] \prod_{k=1}^{m} \lambda_{k}^{n-m} \\
& =\operatorname{det}\left[\begin{array}{ccc}
e^{-a_{1} \lambda_{1}} & \cdots & e^{-a_{1} \lambda_{m}} \\
\vdots & \vdots & \vdots \\
e^{-a_{m} \lambda_{1}} & \cdots & e^{-a_{m} \lambda_{m}}
\end{array}\right] \operatorname{det}\left[\begin{array}{ccc}
\lambda_{1}^{n-m} & \cdots & \lambda_{m}^{n-m} \\
\vdots & \vdots & \vdots \\
\lambda_{1}^{n-1} & \cdots & \lambda_{m}^{n-1}
\end{array}\right] \\
& =\left\{\widetilde{\sum_{i}}(-1)^{\operatorname{per}\left(i_{1}, \cdots, i_{m}\right)} \exp \left(\sum_{j=1}^{m}-a_{i_{j}} \lambda_{j}\right)\right\} \\
& \times\left\{\widetilde{\sum_{k}}(-1)^{\operatorname{per}\left(k_{1}, \cdots, k_{m}\right)} \prod_{l=1}^{m} \lambda_{l}^{n-m+k_{l}}\right\} .
\end{aligned}
$$

The result follows.

Note that, if $\Sigma=\sigma^{2} I_{m}$, then the joint density of the eigenvalues $\lambda_{1}, \ldots, \lambda_{m}$ has a simple form and does not require a zonal polynomial representation.

Proposition 3. Let $\mathbf{W} \sim \mathcal{C} W_{m}\left(n, \sigma^{2} I_{m}\right)$ with $n>m-1$. Then the joint density of the eigenvalues, $\lambda_{1}>\lambda_{2}>\cdots>\lambda_{m}>0$, of $\mathbf{W}$ is

$$
g(\Lambda)=\frac{\pi^{m(m-1)}\left(\sigma^{2}\right)^{-n m}}{\mathcal{C} \Gamma_{m}(m) \mathcal{C} \Gamma_{m}(n)} \prod_{k=1}^{m} \lambda_{k}^{n-m} \prod_{k<l}^{m}\left(\lambda_{k}-\lambda_{l}\right)^{2} \exp \left(-\frac{1}{\sigma^{2}} \sum_{k=1}^{m} \lambda_{k}\right),
$$

where $\Lambda=\operatorname{diag}\left(\lambda_{1}, \ldots, \lambda_{m}\right)$.

Proof. Putting $\Sigma=\sigma^{2} I_{m}$ in Proposition 2 and noting that

$$
\begin{aligned}
\int_{U(m)} \operatorname{etr}\left(-\frac{1}{\sigma^{2}} E \Lambda E^{H}\right)(d E) & =\operatorname{etr}\left(-\frac{1}{\sigma^{2}} \Lambda\right) \int_{U(m)}(d E) \\
& =\exp \left(-\frac{1}{\sigma^{2}} \sum_{i=1}^{m} \lambda_{i}\right)
\end{aligned}
$$


completes the proof.

The single unordered eigenvalue density $f\left(\lambda_{1}\right)$ of $\mathbf{W}$ is given by the following theorem.

TheOREM 2. Let $\mathbf{W} \sim \mathcal{C} W_{m}\left(n, \sigma^{2} I_{m}\right)$ with $n>m-1$. Then the single unordered eigenvalue density $f\left(\lambda_{1}\right)$ of $\mathbf{W}$ is given by

$$
\begin{aligned}
f\left(\lambda_{1}\right)= & \frac{\pi^{m(m-1)}\left(\sigma^{2}\right)^{-n m}}{m ! \mathcal{C} \Gamma_{m}(m) \mathcal{C} \Gamma_{m}(n)} \\
& \times \int \prod_{k=1}^{m} \lambda_{k}^{n-m} \prod_{k<l}^{m}\left(\lambda_{k}-\lambda_{l}\right)^{2} \exp \left(-\frac{1}{\sigma^{2}} \sum_{k=1}^{m} \lambda_{k}\right) \bigwedge_{k=2}^{m} d \lambda_{k} \\
= & \frac{1}{m} \sum_{k=1}^{m}\left[\varphi_{k}\left(\lambda_{1}\right)\right]^{2},
\end{aligned}
$$

where $\varphi_{k}$ form the orthonormal set which can be obtained by applying the GramSchmidt procedure to the sequence of functions

$$
\lambda^{k+(n-m) / 2} e^{-\lambda /\left(2 \sigma^{2}\right)}, \quad k=0,1,2, \ldots, m-1 .
$$

Proof. See [19] for similar work. The joint eigenvalue distribution can be written as

$$
\begin{aligned}
& f(\Lambda)=\frac{K}{m !} \prod_{k<l}^{m}\left(\lambda_{k}-\lambda_{l}\right)^{2}\left[\prod_{k=1}^{m} \lambda_{k}^{n-m} \exp \left(-\frac{1}{\sigma^{2}} \lambda_{k}\right)\right] \\
& =\frac{K}{m !}\left\{\operatorname{det}\left[\begin{array}{ccc}
1 & \cdots & 1 \\
\lambda_{1} & \cdots & \lambda_{n_{t}} \\
\vdots & & \vdots \\
\lambda_{1}^{m-1} & \ldots & \lambda_{m}^{m-1}
\end{array}\right]\right\}^{2}\left[\prod_{k=1}^{m} \lambda_{k}^{n-m} \exp \left(-\frac{1}{\sigma^{2}} \lambda_{k}\right)\right] \\
& =\frac{K}{m !}\left\{\operatorname{det}\left[\begin{array}{ccc}
\lambda_{1}^{(n-m) / 2} e^{-\frac{\lambda_{1}}{2 \sigma^{2}}} & \cdots & \lambda_{m}^{(n-m) / 2} e^{-\frac{\lambda_{m}}{2 \sigma^{2}}} \\
\vdots & & \vdots \\
\lambda_{1}^{(n+m) / 2-1} e^{-\frac{\lambda_{1}}{2 \sigma^{2}}} & \cdots & \lambda_{m}^{(n+m) / 2-1} e^{-\frac{\lambda_{m}}{2 \sigma^{2}}}
\end{array}\right]\right\}^{2} \\
& =\frac{K_{1}}{m !}\left\{\operatorname{det}\left[\begin{array}{ccc}
\varphi_{1}\left(\lambda_{1}\right) & \cdots & \varphi_{1}\left(\lambda_{n_{t}}\right) \\
\vdots & & \vdots \\
\varphi_{m}\left(\lambda_{1}\right) & \cdots & \varphi_{m}\left(\lambda_{m}\right)
\end{array}\right]\right\}^{2}
\end{aligned}
$$

where $\varphi_{k}$ is defined in Theorem 2 and satisfies

$$
\int \varphi_{k}(\lambda) \varphi_{l}(\lambda) d \lambda=\delta_{k l}
$$

The last determinant squared in (16) can be expanded as

$$
\widetilde{\sum_{r, s}}(-1)^{\operatorname{per}\left(r_{1}, \ldots, r_{m}\right)}(-1)^{\operatorname{per}\left(s_{1}, \ldots, s_{m}\right)} \prod_{k} \varphi_{r_{k}}\left(\lambda_{k}\right) \varphi_{s_{k}}\left(\lambda_{k}\right)
$$


where $\widetilde{\sum}_{r, s}$ denotes summation over all permutations $\left(r_{1}, \ldots, r_{m}\right)$ and $\left(s_{1}, \ldots, s_{m}\right)$ of $(1, \ldots, m)$ and $\operatorname{per}\left(r_{1}, \ldots, r_{m}\right)$ is 0 or 1 depending on the permutation being even or odd. Similarly for $\operatorname{per}\left(s_{1}, \ldots, s_{m}\right)$. Hence, $f\left(\lambda_{1}\right)$ can be obtained by integrating (16) with respect to $\lambda_{2}, \ldots, \lambda_{m}$, i.e.,

$$
\begin{aligned}
& f\left(\lambda_{1}\right)=\int f(\Lambda) \bigwedge_{k=2}^{m} d \lambda_{k} \\
& =\frac{K_{1}}{m !} \sum_{r, s}(-1)^{\operatorname{per}\left(r_{1}, \ldots, r_{m}\right)}(-1)^{\operatorname{per}\left(s_{1}, \ldots, s_{m}\right)} \\
& \times \int \prod_{k} \varphi_{r_{k}}\left(\lambda_{k}\right) \varphi_{s_{k}}\left(\lambda_{k}\right) \bigwedge_{k=2}^{m} d \lambda_{k} \\
& =\frac{K_{1}}{m !} \widetilde{\sum_{r, s}}(-1)^{\operatorname{per}\left(r_{1}, \ldots, r_{m}\right)}(-1)^{\operatorname{per}\left(s_{1}, \ldots, s_{m}\right)} \varphi_{r_{1}}\left(\lambda_{1}\right) \varphi_{s_{1}}\left(\lambda_{1}\right) \prod_{k \geq 2}^{m} \delta_{r_{k} s_{k}} \\
& =\frac{K_{1}(m-1) !}{m !} \sum_{k=1}^{m}\left[\varphi_{k}\left(\lambda_{1}\right)\right]^{2} \\
& =\frac{1}{m} \sum_{k=1}^{m}\left[\varphi_{k}\left(\lambda_{1}\right)\right]^{2} \text {. }
\end{aligned}
$$

Since $\int f\left(\lambda_{1}\right) d \lambda_{1}=1$, then $K_{1}=1$.

REMARK 2. The evaluation of (15) for $\sigma^{2}=1$ is given in [3], [12] and [18], where the following formula

$$
f\left(\lambda_{1}\right)=\frac{1}{m} \sum_{l=1}^{m}\left[\varphi_{l}\left(\lambda_{1}\right)\right]^{2}
$$

is obtained with

$$
\begin{aligned}
\varphi_{k+1}(\lambda) & =\lambda^{(n-m) / 2} e^{-\lambda / 2}\left[\frac{k !}{(k+n-m) !}\right]^{1 / 2} \frac{1}{k !} e^{\lambda} \lambda^{m-n} \frac{d^{k}}{d \lambda^{k}}\left(e^{-\lambda} \lambda^{n-m+k}\right) \\
& =\lambda^{(n-m) / 2} e^{-\lambda / 2}\left[\frac{k !}{(k+n-m) !}\right]^{1 / 2} L_{k}^{n-m}(\lambda), \quad k=0, \ldots, m-1,
\end{aligned}
$$

and $L_{k}^{n-m}(\lambda)$ is the generalized Laguerre polynomial of order $k$.

4. The channel capacity. A MIMO channel can be represented by an $n_{r} \times n_{t}$ complex random matrix $\mathbf{H}$, where $n_{t}$ and $n_{r}$ are the number of inputs (or transmitters) and outputs (or receivers) of the communication system, as shown in Figure 1. The complex signal received at the $j$ th output can be written as

$$
y_{j}=\sum_{i=1}^{n_{t}} h_{i j} x_{i}+v_{j}
$$

where $h_{i j}$ is the complex channel coefficient between input $i$ and output $j, x_{i}$ is the complex signal at the $i$ th input and $v_{j}$ is complex Gaussian noise. The signal vector 
received at the output can be written as

$$
\left[\begin{array}{c}
y_{1} \\
\vdots \\
y_{n_{r}}
\end{array}\right]=\left[\begin{array}{ccc}
h_{11} & \cdots & h_{n_{t} 1} \\
\vdots & \vdots & \vdots \\
h_{1 n_{r}} & \cdots & h_{n_{t} n_{r}}
\end{array}\right]\left[\begin{array}{c}
x_{1} \\
\vdots \\
x_{n_{t}}
\end{array}\right]+\left[\begin{array}{c}
v_{1} \\
\vdots \\
v_{n_{r}}
\end{array}\right],
$$

or, in vector notation,

$$
y=H x+v
$$

where $y, v \in \mathbb{C}^{n_{r}}, H \in \mathbb{C}^{n_{r} \times n_{t}}$, and $x \in \mathbb{C}^{n_{t}}$. The total power of the input is constrained to $\rho$,

$$
\mathcal{E}\left\{x^{H} x\right\} \leq \rho \quad \text { or } \quad \operatorname{tr} \mathcal{E}\left\{x x^{H}\right\} \leq \rho .
$$

We shall deal exclusively with the linear model (19) and derive the capacity of MIMO channel models in this section. In this work, we are particularly interested in Rayleigh

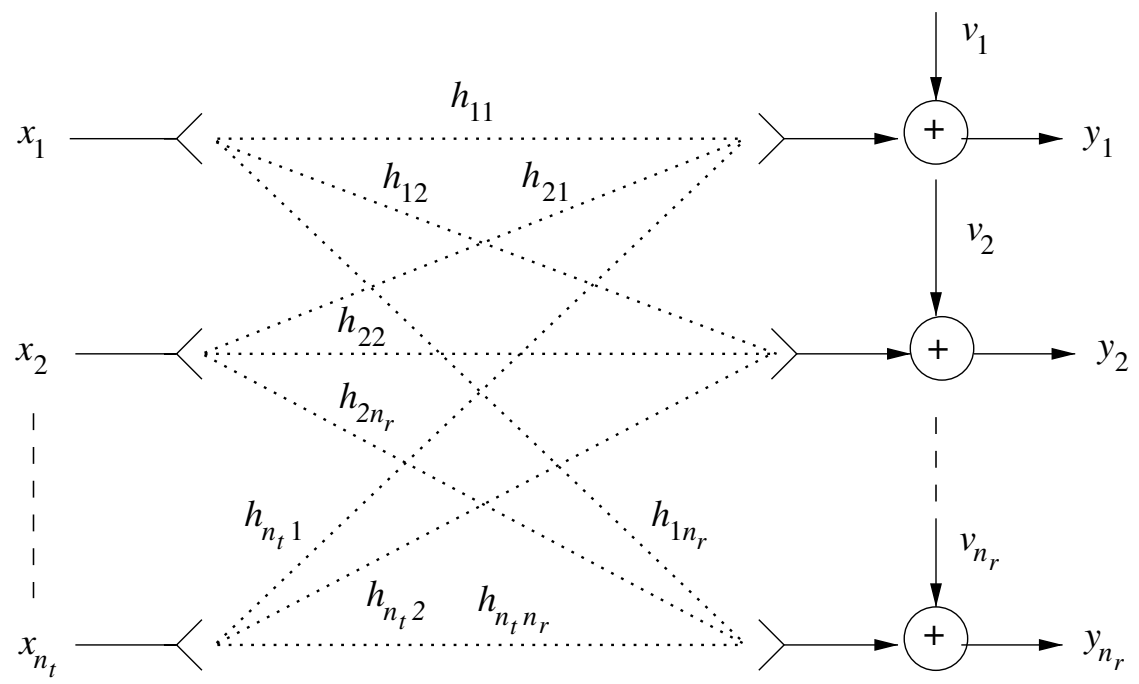

FIG. 1. A MIMO communication system.

distributed channels. The following proposition defines this channel model [13].

Proposition 4. Let $z=r e^{i \theta}\left(=h_{i j}\right) \sim \mathcal{C} N\left(0, \sigma^{2}\right)$, where $r=|z|$ and $\theta=\arg z$. Moreover, we have

$$
\operatorname{var}\{z\}=\mathcal{E}|z|^{2}=\sigma^{2} \quad \text { and } \quad f(z)=\frac{1}{\pi \sigma^{2}} \exp \left(\frac{-|z|^{2}}{\sigma^{2}}\right) .
$$

The density of the magnitude or envelope $r$ is called the Rayleigh density and is given by

$$
h\left(r \mid \sigma^{2}\right)= \begin{cases}\frac{2 r}{\sigma^{2}} \exp \left(\frac{-r^{2}}{\sigma^{2}}\right) & r \geq 0, \\ 0 & r<0 .\end{cases}
$$


The distribution of the phase $\theta$ is uniform and its density is given by

$$
k\left(\theta \mid \sigma^{2}\right)= \begin{cases}\frac{1}{2 \pi} & 0 \leq \theta<2 \pi \\ 0 & \text { otherwise }\end{cases}
$$

We assume that $\mathbf{H}$ is a complex Gaussian random matrix whose realization is known to the receiver, or equivalently, the channel output consists of the pair $(y, \mathbf{H})$. The input power is distributed equally over all transmitting antennas. Moreover, if we assume a block-fading model and coding over many independent fading intervals, then the Shannon or ergodic capacity of the random MIMO channel is given in [18] by

$$
C=\mathcal{E}_{\mathbf{H}}\left\{\log \operatorname{det}\left(I_{n_{t}}+\frac{\rho}{n_{t}} \mathbf{H}^{H} \mathbf{H}\right)\right\},
$$

where the expectation is evaluated using a complex Gaussian density. By Proposition 4, if $\mathbf{H} \sim \mathcal{C} N\left(\mathbf{0}, I_{n_{r}} \otimes \Sigma\right)$ then the channel is Rayleigh distributed. This is typical of fixed or mobile communication environments.

In the calculation of capacity we assume $n_{r} \geq n_{t}$. In this case, the distribution of the channel matrix is given by $\mathbf{H} \sim \mathcal{C} N\left(\mathbf{0}, I_{n_{r}} \otimes \Sigma\right)$. Therefore, the distribution of an $n_{t} \times n_{t}$ complex Wishart matrix is given by $\mathbf{W}=\mathbf{H}^{H} \mathbf{H} \sim \mathcal{C} W_{n_{t}}\left(n_{r}, \Sigma\right)$. Here the covariance matrix of the rows of $\mathbf{H}$ is denoted by $\Sigma$, which is an $n_{t} \times n_{t}$ Hermitian matrix.

5. Computation of the capacity. In a correlated Rayleigh channel, the distribution of an $n_{r} \times n_{t}$ channel matrix $\mathbf{H}$ is given by $\mathbf{H} \sim \mathcal{C} N\left(\mathbf{0}, I_{n_{r}} \otimes \Sigma\right)$, with $n_{r} \geq n_{t}$. Note that the off-diagonal elements of an $n_{t} \times n_{t}$ Hermitian matrix $\Sigma$ are nonzero for correlated channels. In other words, the channel coefficient from different transmitter antennas to a single receiver antenna is correlated. The following lemma is required in the sequel.

LEMMA 1. If $X$ is an $n \times m(n \geq m)$ full rank matrix and the function $f(X)$ depends on $X$ through $X^{H} X$, then

$$
\int_{X^{H} X=A} f\left(X^{H} X\right)(d X)=\frac{\pi^{n m}}{\mathcal{C} \Gamma_{m}(n)}(\operatorname{det} A)^{n-m} f(A) .
$$

Proof. Since $X^{H} X=A$, we have

$$
\int_{X^{H} X=A} f\left(X^{H} X\right)(d X)=f(A) \int_{X^{H} X=A}(d X) .
$$

Let $X=E T$ and $A=T^{H} T$, where $E^{H} E=I_{m}$ and $T$ is an upper triangular matrix with real and positive diagonal elements. Then, from [15, Theorems 4 and 2] we have the following Jacobians for the change of variables

$$
(d X)=\prod_{k=1}^{m} t_{k k}^{2 n-2 k+1}(d T)\left(E^{H} d E\right)
$$


and

$$
(d A)=2^{m} \prod_{k=1}^{m} t_{k k}^{2 m-2 k+1}(d T) .
$$

Hence

$$
(d X)=2^{-m} \prod_{k=1}^{m} t_{k k}^{2 n-2 m}(d A)\left(E^{H} d E\right) .
$$

Moreover, we have

$$
\prod_{k=1}^{m} t_{k k}=\operatorname{det} T=\left(\operatorname{det} T^{H} T\right)^{1 / 2}=(\operatorname{det} A)^{1 / 2} .
$$

Therefore,

$$
\begin{aligned}
f(A) \int_{X^{H} X=A}(d X) & =2^{-m} f(A)(\operatorname{det} A)^{n-m} \int_{\mathcal{C} V_{m, n}}\left(E^{H} d E\right) \\
& =\frac{\pi^{n m}}{\mathcal{C} \Gamma_{m}(n)}(\operatorname{det} A)^{n-m} f(A) .
\end{aligned}
$$

The last equality follows from [15, Theorem 5].

The channel capacity is given by the following theorem. We shall assume that the realization of $\mathbf{H}$ is known to the receiver, or equivalently, the channel output consists of the pair $(y, \mathbf{H})$.

TheOREM 3. Consider the correlated Rayleigh channel, i.e., $\mathbf{H} \sim \mathcal{C} N\left(\mathbf{0}, I_{n_{r}} \otimes \Sigma\right)$, with $n_{r} \geq n_{t}$. If the input power is constrained by $\rho$, then the capacity $C$ is given by

$$
\frac{1}{\mathcal{C} \Gamma_{n_{t}}\left(n_{r}\right)(\operatorname{det} \Sigma)^{n_{r}}} \int_{W>0} \log \operatorname{det}\left[I_{n_{t}}+\frac{\rho}{n_{t}} W\right](\operatorname{det} W)^{n_{r}-n_{t}} \operatorname{etr}\left(-\Sigma^{-1} W\right)(d W),
$$

where $W=H^{H} H$.

Proof. From formula (22), the capacity $C$ is given by

$$
C=\int_{H} \log \operatorname{det}\left[I_{n_{t}}+\frac{\rho}{n_{t}} H^{H} H\right] f(H)(d H),
$$

where

$$
f(H)=\pi^{-n_{r} n_{t}}(\operatorname{det} \Sigma)^{-n_{r}} \operatorname{etr}\left(-\Sigma^{-1} H^{H} H\right) .
$$

Using Lemma 1, we can write $C$ as

$$
\begin{aligned}
C= & \pi^{-n_{r} n_{t}}(\operatorname{det} \Sigma)^{-n_{r}} \int_{H} \log \operatorname{det}\left[I_{n_{t}}+\frac{\rho}{n_{t}} H^{H} H\right] \operatorname{etr}\left(-\Sigma^{-1} H^{H} H\right)(d H) \\
= & \pi^{-n_{r} n_{t}}(\operatorname{det} \Sigma)^{-n_{r}} \\
& \times \int_{W>0} \int_{H^{H} H=W} \log \operatorname{det}\left[I_{n_{t}}+\frac{\rho}{n_{t}} H^{H} H\right] \operatorname{etr}\left(-\Sigma^{-1} H^{H} H\right)(d H)(d W) \\
= & \frac{1}{\mathcal{C} \Gamma_{n_{t}}\left(n_{r}\right)(\operatorname{det} \Sigma)^{n_{r}}} \\
& \times \int_{W>0} \log \operatorname{det}\left[I_{n_{t}}+\frac{\rho}{n_{t}} W\right](\operatorname{det} W)^{n_{r}-n_{t}} \operatorname{etr}\left(-\Sigma^{-1} W\right)(d W) .
\end{aligned}
$$


This completes the proof.

Theorem 3 can also be obtained by using (22) and the complex central Wishart density given in (6). Now, using the eigenvalue density of a complex central Wishart matrix, the correlated Rayleigh channel capacity can be expressed as follows.

THEOREM 4. Consider the correlated Rayleigh channel, i.e., $\mathbf{H} \sim \mathcal{C} N\left(\mathbf{0}, I_{n_{r}} \otimes \Sigma\right)$, with $n_{r} \geq n_{t}$. If the input power is constrained by $\rho$, then using the joint eigenvalue density of the complex central Wishart matrix $\mathbf{W}=\mathbf{H}^{H} \mathbf{H}$ we can write the capacity $C$ as

$$
K \int_{\Lambda>0} \log \left\{\prod_{k=1}^{n_{t}}\left[1+\frac{\rho}{n_{t}} \lambda_{k}\right]\right\} \prod_{k=1}^{n_{t}} \lambda_{k}^{n_{r}-n_{t}} \prod_{k<l}^{n_{t}}\left(\lambda_{k}-\lambda_{l}\right)^{2}{ }_{0} F_{0}\left(-\Sigma^{-1}, \Lambda\right) \bigwedge_{k=1}^{n_{t}} d \lambda_{k},
$$

where $\lambda_{1}>\cdots>\lambda_{n_{t}}>0$ are the eigenvalues of $\mathbf{W}, \Lambda=\operatorname{diag}\left(\lambda_{1}, \ldots, \lambda_{n_{t}}\right)$, and

$$
K=\frac{\pi^{n_{t}\left(n_{t}-1\right)}(\operatorname{det} \Sigma)^{-n_{r}}}{\mathcal{C} \Gamma_{n_{t}}\left(n_{t}\right) \mathcal{C} \Gamma_{n_{t}}\left(n_{r}\right)}
$$

Proof. From Theorem 3, the capacity $C$ is given by

$$
C=\mathcal{E}_{\mathbf{W}}\left\{\log \operatorname{det}\left(I_{n_{t}}+\frac{\rho}{n_{t}} \mathbf{W}\right)\right\}=\mathcal{E}_{\Lambda}\left\{\log \left(\prod_{k=1}^{n_{t}}\left[1+\frac{\rho}{n_{t}} \lambda_{k}\right]\right)\right\} .
$$

The result follows by using the following joint eigenvalue density (see Propositions 2),

$$
f\left(\lambda_{1}, \ldots, \lambda_{n_{t}}\right)=\frac{\pi^{n_{t}\left(n_{t}-1\right)}(\operatorname{det} \Sigma)^{-n_{r}}}{\mathcal{C} \Gamma_{n_{t}}\left(n_{t}\right) \mathcal{C} \Gamma_{n_{t}}\left(n_{r}\right)} \prod_{k=1}^{n_{t}} \lambda_{k}^{n_{r}-n_{t}} \prod_{k<l}^{n_{t}}\left(\lambda_{k}-\lambda_{l}\right)^{2}{ }_{0} F_{0}\left(-\Sigma^{-1}, \Lambda\right) .
$$

The proof is complete.

TheOrem 5. Consider the correlated Rayleigh channel, i.e., $\mathbf{H} \sim \mathcal{C} N\left(\mathbf{0}, I_{n_{r}} \otimes \Sigma\right)$, with $n_{r} \geq n_{t}$. If the input power is constrained by $\rho$, then using the single unordered eigenvalue density we can write the capacity $C$ as

$$
C=n_{t} \mathcal{E}_{\lambda_{1}}\left[\log \left(1+\left(\rho / n_{t}\right) \lambda_{1}\right)\right]
$$

The density $f\left(\lambda_{1}\right)$ is given by

$$
\begin{aligned}
& f\left(\lambda_{1}\right)=\frac{\pi^{n_{t}\left(n_{t}-1\right) / 2} \prod_{k=1}^{n_{t}} a_{k}^{n_{r}}}{n_{t} ! \mathcal{C} \Gamma_{n_{t}}\left(n_{r}\right) \prod_{k<l}^{n_{t}}\left(a_{l}-a_{k}\right)} \\
& \times \int\left\{\widetilde{\sum_{i}}(-1)^{\operatorname{per}\left(i_{1}, \ldots, i_{n_{t}}\right)} \exp \left(\sum_{j=1}^{n_{t}}-a_{i_{j}} \lambda_{j}\right)\right\} \\
& \times\left\{\widetilde{\sum_{k}}(-1)^{\operatorname{per}\left(k_{1}, \ldots, k_{n_{t}}\right)} \prod_{l=1}^{n_{t}} \lambda_{l}^{n_{r}-n_{t}+k_{l}}\right\} \bigwedge_{k=2}^{n_{t}} d \lambda_{k},
\end{aligned}
$$


where $\widetilde{\sum}_{i}$ denotes summation over all permutations $\left(i_{1}, \ldots, i_{n_{t}}\right)$ of $\left(1, \ldots, n_{t}\right), \widetilde{\sum}_{k} d e$ notes summation over all permutations $\left(k_{1}, \ldots, k_{n_{t}}\right)$ of $\left(0, \ldots, n_{t}-1\right)$ and $\operatorname{per}\left(k_{1}, \ldots\right.$, $\left.k_{n_{t}}\right)$ is 0 or 1 depending on the permutation being even or odd. Similarly for $\operatorname{per}\left(i_{1}, \ldots\right.$, $\left.i_{n_{t}}\right)$. Note that $\left(a_{1}, \ldots, a_{n_{t}}\right)$ are eigenvalues of $\Sigma^{-1}$.

Proof. From (24), $C$ can be written as

$$
C=\sum_{k=1}^{n_{t}} \mathcal{E}_{\lambda_{k}}\left[\log \left(1+\frac{\rho}{n_{t}} \lambda_{k}\right)\right]=n_{t} \mathcal{E}_{\lambda_{1}}\left[\log \left(1+\frac{\rho}{n_{t}} \lambda_{1}\right)\right],
$$

where the expectation is with respect to $\lambda_{1}$. Because we are using the single unordered eigenvalue density (12), the result follows.

5.1. Correlated Rayleigh $n_{r} \times 2$ channel matrix. In this subsection, a numerical evaluation of a correlated Rayleigh $n_{r} \times 2$ channel matrix is given. Thus, we assume that we have a two-input $\left(n_{t}=2\right), n_{r}$-output communication system operating over a correlated Rayleigh fading environment (typical mobile wireless environment). As mentioned before, the joint eigenvalue density of a central Wishart matrix depends on the population covariance matrix $\Sigma$ only through its eigenvalues $v_{1}, \ldots, v_{n_{t}}$, i.e.,

$$
{ }_{0} F_{0}\left(-\Sigma^{-1}, \Lambda\right)={ }_{0} F_{0}\left(-\Upsilon^{-1}, \Lambda\right),
$$

where $\Upsilon=\operatorname{diag}\left(v_{1}, \ldots, v_{n_{t}}\right)$. Let $n_{t}=2$ and $\Upsilon^{-1}=\operatorname{diag}\left(a_{1}, a_{2}\right)$. Then we have [10]

$$
\begin{aligned}
{ }_{0} F_{0}\left(-\Upsilon^{-1}, \Lambda\right)= & \frac{1}{\left(a_{2}-a_{1}\right)\left(\lambda_{1}-\lambda_{2}\right)} \\
& \times\left[\exp \left\{-\left(a_{1} \lambda_{1}+a_{2} \lambda_{2}\right)\right\}-\exp \left\{-\left(a_{1} \lambda_{2}+a_{2} \lambda_{1}\right)\right\}\right] .
\end{aligned}
$$

The following theorem gives the correlated Rayleigh channel capacity for an $n_{r} \times 2$ matrix.

TheOREM 6. Consider a two-input correlated Rayleigh channel, $\mathbf{H} \sim \mathcal{C} N\left(\mathbf{0}, I_{n_{r}} \otimes\right.$ $\Sigma)$, with $n_{r} \geq 2$. If the input power is constrained by $\rho$, then the capacity $C$ is

$$
\begin{aligned}
C= & \frac{a_{1}^{n_{r}} a_{2}}{\left(a_{2}-a_{1}\right) \Gamma\left(n_{r}\right)} \int_{0}^{\infty} \log \left[1+\frac{\rho}{2} \lambda_{1}\right] \lambda_{1}^{n_{r}-1} e^{-a_{1} \lambda_{1}} d \lambda_{1} \\
& -\frac{a_{1} a_{2}^{n_{r}}}{\left(a_{2}-a_{1}\right) \Gamma\left(n_{r}\right)} \int_{0}^{\infty} \log \left[1+\frac{\rho}{2} \lambda_{1}\right] \lambda_{1}^{n_{r}-1} e^{-a_{2} \lambda_{1}} d \lambda_{1} \\
& -\frac{a_{1}^{n_{r}}}{\left(a_{2}-a_{1}\right) \Gamma\left(n_{r}-1\right)} \int_{0}^{\infty} \log \left[1+\frac{\rho}{2} \lambda_{1}\right] \lambda_{1}^{n_{r}-2} e^{-a_{1} \lambda_{1}} d \lambda_{1} \\
& +\frac{a_{2}^{n_{r}}}{\left(a_{2}-a_{1}\right) \Gamma\left(n_{r}-1\right)} \int_{0}^{\infty} \log \left[1+\frac{\rho}{2} \lambda_{1}\right] \lambda_{1}^{n_{r}-2} e^{-a_{2} \lambda_{1}} d \lambda_{1},
\end{aligned}
$$

where $\lambda_{1}$ is an unordered eigenvalue of $W=H^{H} H$ and $\left(a_{1}, a_{2}\right)$ are eigenvalues of $\Sigma^{-1}$.

Proof. By (28), the unordered eigenvalue density of $W$ is given by

$$
f\left(\lambda_{1}, \lambda_{2}\right)=\frac{\left(a_{1} a_{2}\right)^{n_{r}}\left(\lambda_{1} \lambda_{2}\right)^{n_{r}-2}\left(\lambda_{1}-\lambda_{2}\right)}{2\left(a_{2}-a_{1}\right) \Gamma\left(n_{r}\right) \Gamma\left(n_{r}-1\right)}\left[e^{-a_{1} \lambda_{1}-a_{2} \lambda_{2}}-e^{-a_{1} \lambda_{2}-a_{2} \lambda_{1}}\right] .
$$


Now, integrating with respect to $\lambda_{2}$ and noting that

$$
\int_{0}^{\infty} x^{a-1} e^{-x / b} d x=\Gamma(a) b^{a}
$$

we obtain the density of $\lambda_{1}$,

$$
\begin{aligned}
f\left(\lambda_{1}\right)=\frac{1}{2\left(a_{2}-a_{1}\right)}\{ & \frac{a_{1}^{n_{r}} a_{2} \lambda_{1}^{n_{r}-1} e^{-a_{1} \lambda_{1}}}{\Gamma\left(n_{r}\right)}-\frac{a_{1} a_{2}^{n_{r}} \lambda_{1}^{n_{r}-1} e^{-a_{2} \lambda_{1}}}{\Gamma\left(n_{r}\right)} \\
& \left.-\frac{a_{1}^{n_{r}} \lambda_{1}^{n_{r}-2} e^{-a_{1} \lambda_{1}}}{\Gamma\left(n_{r}-1\right)}+\frac{a_{2}^{n_{r}} \lambda_{1}^{n_{r}-2} e^{-a_{2} \lambda_{1}}}{\Gamma\left(n_{r}-1\right)}\right\} .
\end{aligned}
$$

It is easy to see that $\int_{0}^{\infty} f\left(\lambda_{1}\right) d \lambda_{1}=1$. Finally, evaluating (25) with $f\left(\lambda_{1}\right)$ gives (29).

Table 1 shows the capacity in nats ${ }^{1}$ for an $n_{r} \times 2$ correlated Rayleigh fading channel matrix with correlation coefficient 0.9. Note that each column represents different levels of input power or signal-to-noise ratio (SNR) in dB. Figure 2 shows the capacity in nats vs $n_{r}$ for the correlation coefficient 0.9. Figure 3 shows the capacity vs SNR and Figure 4 shows the capacity vs the correlation coefficient. From these tables and figures we note the following: $(i)$ the capacity is decreasing with increasing channel correlation, (ii) the capacity is increasing with increasing $n_{r}$ and SNR.

Note that the covariance matrix is $\Sigma=\left[\begin{array}{ll}1 & 0.9 \\ 0.9 & 1\end{array}\right]$ and its eigenvalues are 1.9 and 0.1. Hence $\Upsilon=\operatorname{diag}(1.9,0.1), a_{1}=1 / 1.9$, and $a_{2}=1 / 0.1$. Note also that the off-diagonal element of $\Sigma$ gives the correlation between the channel coefficient from different transmitter antennas to a single receiver antenna, i.e.,

$$
\mathcal{E}\left\{h_{i j} h_{k l}^{*}\right\}= \begin{cases}0.9 & i \neq k=1,2, j=l=1, \ldots, n_{r}, \\ 0 & \text { otherwise }\end{cases}
$$

This off-diagonal element is called a channel correlation coefficient or correlation coefficient.

5.2. Uncorrelated Rayleigh $n_{r} \times 2$ channel matrix. In this subsection, the numerical evaluation of an uncorrelated Rayleigh $n_{r} \times 2$ channel matrix is given. In other words, we assume we have a two-input $\left(n_{t}=2\right), n_{r}$-output communication system operating over an uncorrelated Rayleigh fading environment, which is a typical fixed wireless environment. The following theorem gives an expression for the capacity $C$.

TheOREm 7. Consider a two-input uncorrelated Rayleigh channel, i.e., $\mathbf{H} \sim$ $\mathcal{C} N\left(\mathbf{0}, I_{n_{r}} \otimes \sigma^{2} I_{2}\right)$, with $n_{r} \geq 2$. If the input power is constrained by $\rho$, then the

\footnotetext{
${ }^{1}$ In (29), if we use $\log _{e}$ then the capacity is measured in nats. If we use $\log _{2}$ then the capacity is measured in bits. Thus, one nat is equal to $e$ bits $/ \mathrm{sec} / \mathrm{Hz}(e=2.718 \ldots)$.
} 
TABLE 1

The capacity in nats for a two-input, $n_{r}$-output communication system operating over a correlated Rayleigh fading channel, where $\rho$ is signal-to-noise ratio in $d B$ and the correlation coefficient is equal to 0.9 .

\begin{tabular}{c|c|c|c|c|c|c|c|c}
\hline & \multicolumn{7}{|c}{$\rho$ in dB } \\
\hline$n_{r}$ & $0 \mathrm{~dB}$ & $5 \mathrm{~dB}$ & $10 \mathrm{~dB}$ & $15 \mathrm{~dB}$ & $20 \mathrm{~dB}$ & $25 \mathrm{~dB}$ & $30 \mathrm{~dB}$ & $35 \mathrm{~dB}$ \\
\hline 2 & 1.0326 & 1.9252 & 3.1157 & 4.5641 & 6.2023 & 7.9419 & 9.7221 & 11.5165 \\
4 & 1.6408 & 2.8426 & 4.4118 & 6.3154 & 8.4439 & 10.6803 & 12.9577 & 15.2490 \\
6 & 2.0685 & 3.4398 & 5.1852 & 7.2250 & 9.4266 & 11.6948 & 13.9863 & 16.2855 \\
8 & 2.4033 & 3.8917 & 5.7454 & 7.8540 & 10.0862 & 12.3653 & 14.6604 & 16.9606 \\
10 & 2.6804 & 4.2568 & 6.1838 & 8.3330 & 10.5817 & 12.8666 & 15.1635 & 17.4643 \\
12 & 2.9179 & 4.5639 & 6.5437 & 8.7196 & 10.9786 & 13.2669 & 15.5650 & 17.8661 \\
14 & 3.1265 & 4.8293 & 6.8489 & 9.0437 & 11.3096 & 13.6003 & 15.8992 & 18.2005 \\
16 & 3.3129 & 5.0631 & 7.1139 & 9.3226 & 11.5936 & 13.8860 & 16.1853 & 18.4869 \\
18 & 3.4817 & 5.2722 & 7.3479 & 9.5674 & 11.8422 & 14.1359 & 16.4357 & 18.7373 \\
20 & 3.6361 & 5.4612 & 7.5574 & 9.7855 & 12.0634 & 14.3580 & 16.6581 & 18.9599 \\
\hline
\end{tabular}

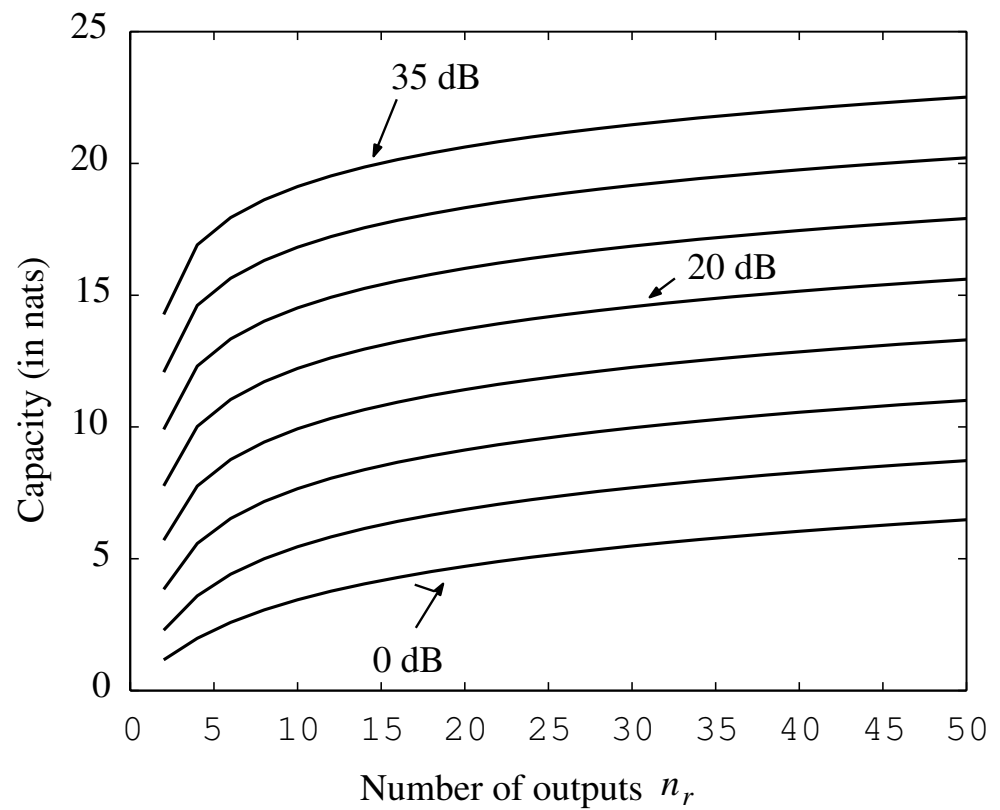

FIG. 2. Capacity vs number of outputs for $\mathrm{SNR}=0,5,10,15,20,25,30,35$ dB. Note that $\mathbf{H}$ is an $n_{r} \times 2$ correlated Rayleigh fading channel matrix with correlation coefficient equal to 0.9 . 


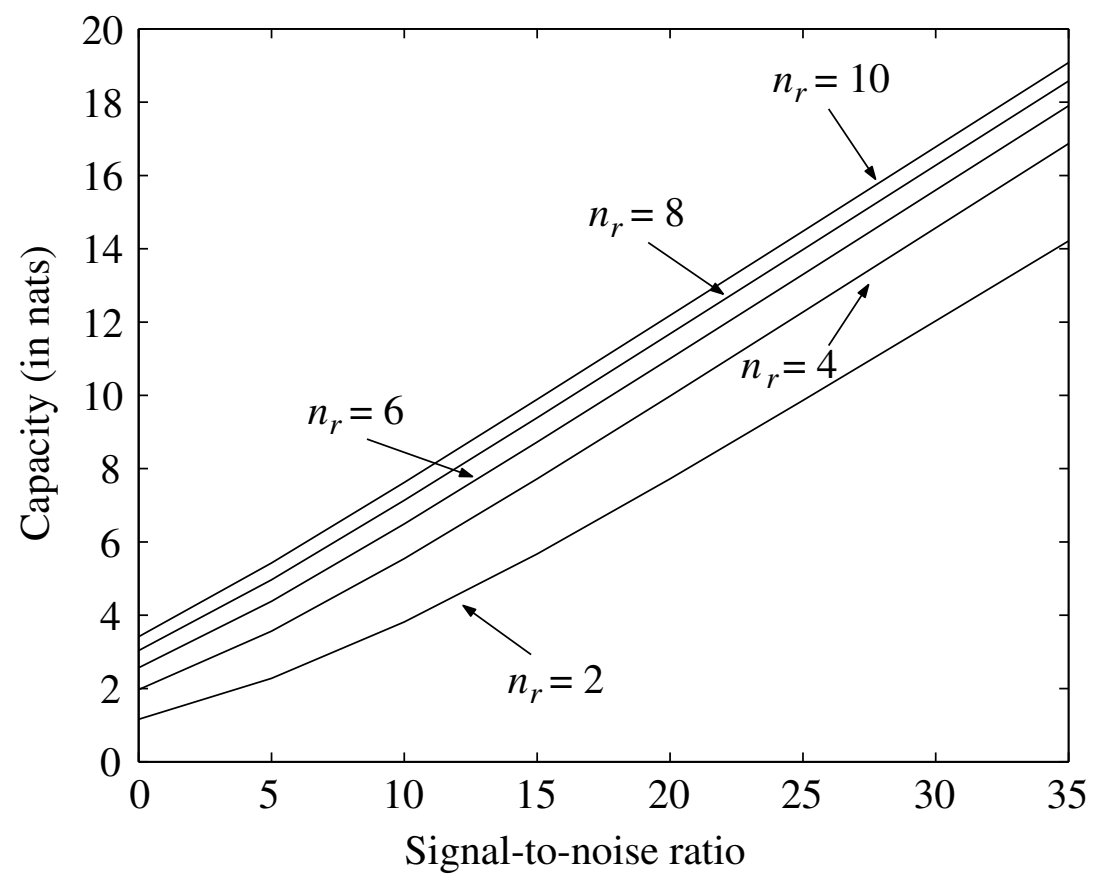

FIG. 3. Capacity vs $S N R$ for correlation coefficient $0.2, n_{t}=2$, and $n_{r}=2,4,6,8,10$, i.e., $\mathbf{H}$ is an $n_{r} \times 2$ correlated Rayleigh fading channel matrix.

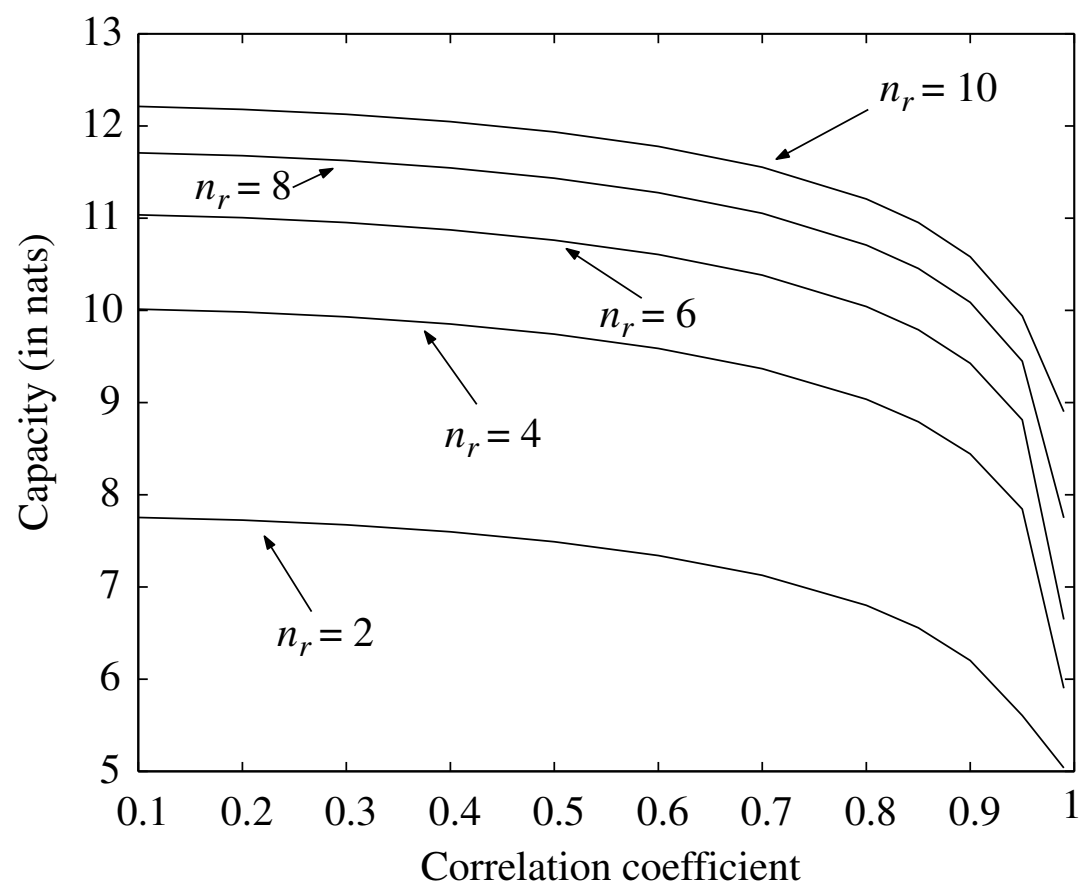

FIG. 4. Capacity vs correlation coefficient for $\mathrm{SNR}=20 \mathrm{~dB}, n_{t}=2$, and $n_{r}=2,4,6,8,10$, i.e., $\mathbf{H}$ is an $n_{r} \times 2$ correlated Rayleigh fading channel matrix. 
capacity $C$ is given by

$$
\begin{aligned}
C= & \frac{\left(\sigma^{2}\right)^{-n_{r}-1}}{\Gamma\left(n_{r}\right)} \int_{0}^{\infty} \log \left[1+\frac{\rho}{2} \lambda_{1}\right] \lambda_{1}^{n_{r}} e^{-\lambda_{1} / \sigma^{2}} d \lambda_{1} \\
& -\frac{2\left(\sigma^{2}\right)^{-n_{r}}}{\Gamma\left(n_{r}-1\right)} \int_{0}^{\infty} \log \left[1+\frac{\rho}{2} \lambda_{1}\right] \lambda_{1}^{n_{r}-1} e^{-\lambda_{1} / \sigma^{2}} d \lambda_{1} \\
& +\frac{\left(\sigma^{2}\right)^{-n_{r}+1} \Gamma\left(n_{r}+1\right)}{\Gamma\left(n_{r}\right) \Gamma\left(n_{r}-1\right)} \int_{0}^{\infty} \log \left[1+\frac{\rho}{2} \lambda_{1}\right] \lambda_{1}^{n_{r}-2} e^{-\lambda_{1} / \sigma^{2}} d \lambda_{1},
\end{aligned}
$$

where $\lambda_{1}$ is an unordered eigenvalue of $W=H^{H} H$.

Proof. By (14), the unordered eigenvalue density of $W$ is

$$
f\left(\lambda_{1}, \lambda_{2}\right)=\frac{\left(\sigma^{2}\right)^{-2 n_{r}}\left(\lambda_{1} \lambda_{2}\right)^{n_{r}-2}\left(\lambda_{1}-\lambda_{2}\right)^{2}}{2 \Gamma\left(n_{r}\right) \Gamma\left(n_{r}-1\right)} e^{-\left(\lambda_{1}+\lambda_{2}\right) / \sigma^{2}} .
$$

Integrating with respect to $\lambda_{2}$ and using (31), we obtain the density of $\lambda_{1}$,

$$
\begin{gathered}
f\left(\lambda_{1}\right)=\frac{\left(\sigma^{2}\right)^{-n_{r}-1}}{2 \Gamma\left(n_{r}\right)} \lambda_{1}^{n_{r}} e^{-\lambda_{1} / \sigma^{2}}-\frac{\left(\sigma^{2}\right)^{-n_{r}}}{\Gamma\left(n_{r}-1\right)} \lambda_{1}^{n_{r}-1} e^{-\lambda_{1} / \sigma^{2}} \\
+\frac{\left(\sigma^{2}\right)^{-n_{r}+1} \Gamma\left(n_{r}+1\right)}{2 \Gamma\left(n_{r}\right) \Gamma\left(n_{r}-1\right)} \lambda_{1}^{n_{r}-2} e^{-\lambda_{1} / \sigma^{2}} .
\end{gathered}
$$

It is easy to see that $\int_{0}^{\infty} f\left(\lambda_{1}\right) d \lambda_{1}=1$. Finally, evaluating (25) with $f\left(\lambda_{1}\right)$ gives (32).

Table 2 shows the capacity in nats for an $n_{r} \times 2$ uncorrelated Rayleigh fading channel matrix with different levels of input power. Figure 5 shows the capacity in nats vs $n_{r}$ for different signal to noise ratios. It is clearly seen from the table and figure that the capacity is increasing with increasing $n_{r}$ and SNR.

6. Conclusion. In this paper, joint and single unordered eigenvalue densities of complex central Wishart matrices are derived. These densities are used to derive formulas for the capacity of correlated and uncorrelated MIMO Rayleigh channels. The capacity of $n_{r} \times 2$ MIMO Rayleigh channel matrices are computed for both correlated and uncorrelated channels. This study shows how the channel correlation degrades the capacity of the communication system. 
TABLE 2

The capacity in nats for a two-input, $n_{r}$-output communication system operating over an uncorrelated Rayleigh fading channel, where $\rho$ is signal-to-noise ratio in $d B$.

\begin{tabular}{c|c|c|c|c|c|c|c|c}
\hline & \multicolumn{7}{|c}{$\rho$ in $\mathrm{dB}$} \\
\hline$n_{r}$ & $0 \mathrm{~dB}$ & $5 \mathrm{~dB}$ & $10 \mathrm{~dB}$ & $15 \mathrm{~dB}$ & $20 \mathrm{~dB}$ & $25 \mathrm{~dB}$ & $30 \mathrm{~dB}$ & $35 \mathrm{~dB}$ \\
\hline 2 & 1.1671 & 2.2890 & 3.8382 & 5.7066 & 7.7633 & 9.9062 & 12.0815 & 14.2676 \\
4 & 1.9831 & 3.5910 & 5.5788 & 7.7614 & 10.0227 & 12.3119 & 14.6102 & 16.9114 \\
6 & 2.5857 & 4.4125 & 6.5274 & 8.7649 & 11.0462 & 13.3420 & 15.6425 & 17.9444 \\
8 & 3.0573 & 5.0020 & 7.1725 & 9.4308 & 11.7191 & 14.0172 & 16.3183 & 18.6204 \\
10 & 3.4425 & 5.4595 & 7.6605 & 9.9296 & 12.2214 & 14.5206 & 16.8221 & 19.1244 \\
12 & 3.7672 & 5.8326 & 8.0528 & 10.3285 & 12.6225 & 14.9223 & 17.2240 & 19.5263 \\
14 & 4.0475 & 6.1475 & 8.3808 & 10.6609 & 12.9563 & 15.2566 & 17.5585 & 19.8608 \\
16 & 4.2939 & 6.4197 & 8.6626 & 10.9458 & 13.2423 & 15.5429 & 17.8449 & 20.1473 \\
18 & 4.5136 & 6.6595 & 8.9096 & 11.1952 & 13.4924 & 15.7933 & 18.0953 & 20.3977 \\
20 & 4.7117 & 6.8736 & 9.1294 & 11.4169 & 13.7147 & 16.0158 & 18.3179 & 20.6203 \\
\hline
\end{tabular}

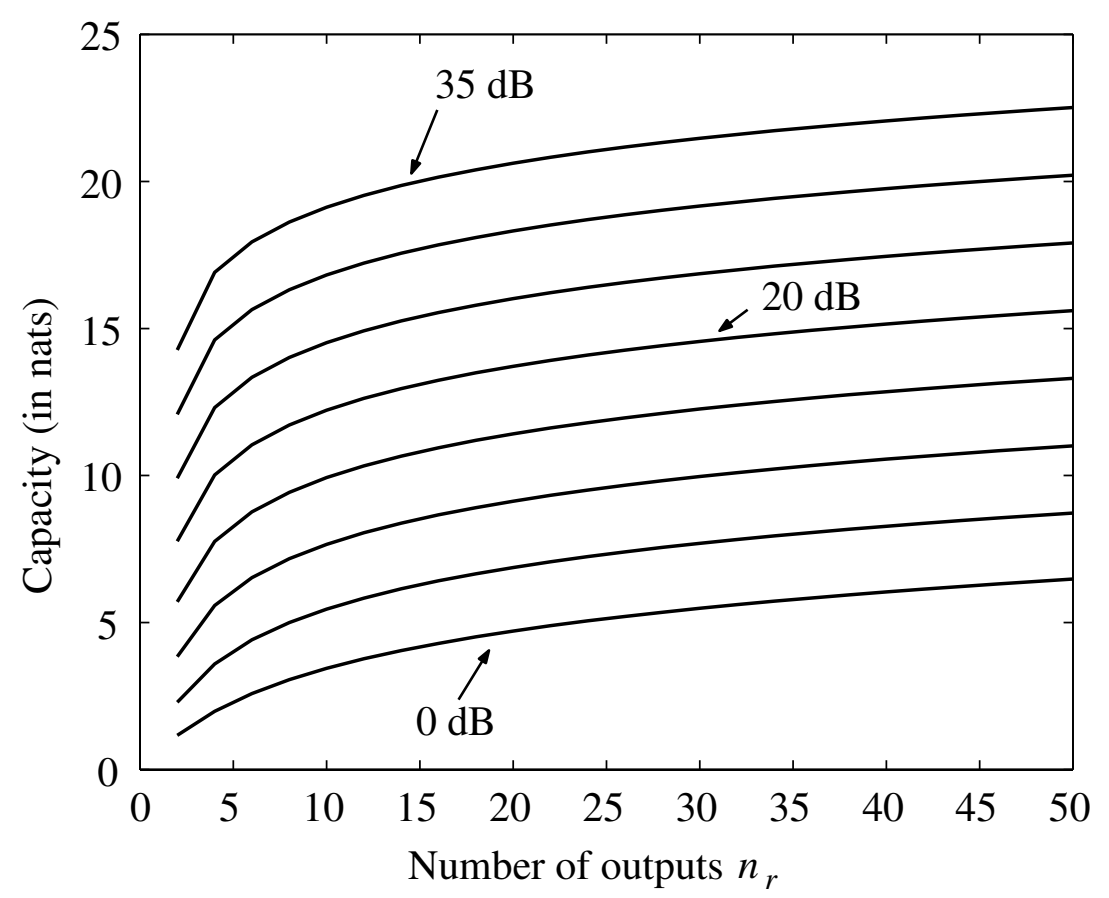

FIG. 5. Capacity vs number of outputs for $\mathrm{SNR}=0,5,10,15,20,25,30,35$ dB. Note that $\mathbf{H}$ is an $n_{r} \times 2$ uncorrelated Rayleigh fading channel matrix. 


\section{REFERENCES}

[1] R. B. Ash, Information Theory, Dover, New York, 1965.

[2] T. Baker and P. Forrester, The Calogero-Sutherland model and generalized classical polynomials, Commun. Math. Phys., 188(1997), pp. 175-216.

[3] B. V. Bronk, Exponential ensembles for random matrices, J. of Math. Physics, 6(1965), pp. 228-237.

[4] C. N. Chuah, D. Tse, J. M. Kahn, and R. A. Valenzuela, Capacity scaling in Mimo wireless systems under correlated fading, IEEE Trans. on Information Theory, 48(2002), pp. $637-650$.

[5] A. G. Constantine, Some noncentral distribution problems in multivariate analysis, Ann. Math. Statist., 34(1963), pp. 1270-1285.

[6] A. Edelman, Eigenvalues and Condition Numbers of Random Matrices, Ph.D. Dissertation, MIT, Cambridge, MA, May 1989.

[7] N. R. Goodman, Statistical analysis based on a certain multivariate complex Gaussian distribution (An introduction), Ann. Math. Statist., 34(1963), pp. 152-177.

[8] A. T. JAmes, Distributions of matrix variate and latent roots derived from normal samples, Ann. Math. Statist., 35(1964), pp. 475-501.

[9] C. G. Khatri, On certain distribution problems based on positive definite quadratic functions in normal vectors, Ann. Math. Statist., 37(1966), pp. 468-479.

[10] C. G. Khatri, Non-central distributions of ith largest characteristic roots of three matrices concerning complex multivariate normal populations, Ann. Inst. Statist. Math., 21(1969), pp. $23-32$.

[11] I. G. Macdonald, Symmetric Functions and Hall Polynomials, Oxford University Press Inc., New York, 1995.

[12] M. L Mehta, Random Matrices, 2nd ed, Academic Press, New York, 1991.

[13] K. S. Miller, Complex Stochastic Processes: An Introduction to Theory and Application, Addison-Wesley, New York, 1974.

[14] R. J. Muirhead, Aspects of Multivariate Statistical Theory, Wiley, New York, 1982.

[15] T. Ratnarajah, R. Vaillancourt, and M. Alvo, Jacobians and Hypergeometric functions in Complex Multivariate Analysis, Can. Appl. Math. Quarterly, to appear.

[16] T. Ratnarajah, R. Vaillancour, and M. Alvo, Complex Random Matrices and Applications", Math. Rep. of the Acad. of Sci. of the Royal Soc. of Canada, to appear.

[17] D. S. Shiu, G. F. Foschini, M. G. Gans, and J. M. Kahn, Fading correlation and its effect on the capacity of multielement antenna systems, IEEE Trans. on Communications, 48(2000), pp. 502-513.

[18] I. E. Telatar, Capacity of multi-antenna Gaussian channels, Eur. Trans. Telecom, 10(1999), pp. 585-595.

[19] E. Wigner, Distribution laws for roots of a random Hermitian matrix, In Statistical Theories of Spectra: Fluctuations, (C. E. Porter, ed.), Academic Press, New York, pp. 446-461, 1965. 